gap> $\mathrm{g}:=$ SymmetricGroup $(4)$;

$\operatorname{Sym}([1 \ldots 4])$

i5 : betti $(t$, Weights $\Rightarrow\{1$, gap $\}$

false

01234 gap> tblmod2:= CharacterTable( tbl, 2);

05 = total: 1413144 BrauerTable $(\operatorname{Sym}([1 \ldots 4]), 2)$

1: . 2242 gap> tblmod2 = CharacterTable $(t b l, 2)$;

Journal of Software for

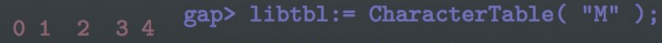

$06=$ total: 1413144 CharacterTable( "M")

fail $\quad r i n g ~ r 1=32003,(x, y, z)$, ds ;

gap> CharacterTable( "Symmetric", 4) ; int a,b,c,t=11,5,3,0;

BettiTally

CharacterTable ( "Sym (4)" )

gap> ComputedBrauerTables ( tbl );

$\begin{array}{lllll}0 & 1 & 2 & 3 & 4\end{array}$

[

poly $f=x^{\wedge} a+y^{\wedge} b+z^{\wedge}(3 * c)+x^{\wedge}(c+2) * y^{\wedge}(c-1)+x^{\wedge}$ $\mathrm{x}^{\sim}(\mathrm{c}-2) * \mathrm{y}^{\wedge} \mathrm{c} *\left(\mathrm{y}^{\sim} 2+\mathrm{t} * \mathrm{x}\right)^{\sim}-2$;

o7 = total: 1413144

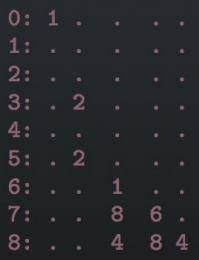

o7 : BettiTally

i8 : peek t1

$08=$ BettiTally $\{(0,\{0,0\}, 0) \Rightarrow 1\}$

$(1,\{2,2\}, 4) \Rightarrow 2$

$(1,\{3,3\}, 6) \Rightarrow 2$

(2, $\{3,7\}, 10) \Rightarrow 2$

$(2,\{4,4\}, 8) \Rightarrow 1$

(2, $\{4,5\}, 9) \Rightarrow 4$

$(2,\{5,4\}, 9) \Rightarrow 4$

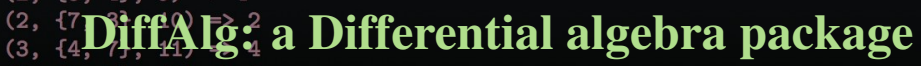

$(3,\{5,5\}, 10) \Rightarrow 6$

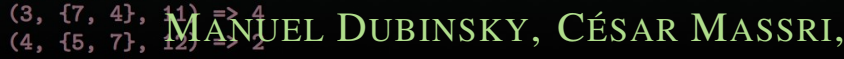

(4. AR PËL ${ }^{1 M}$ MOLINUEVO AND FEDERICO QUALLBRUNN

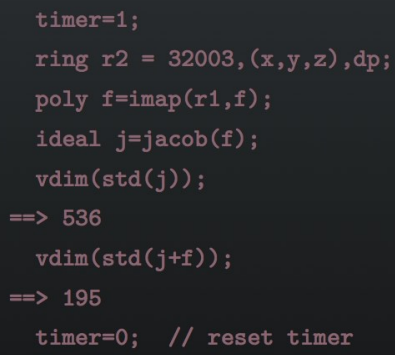




\title{
DiffAlg: a Differential algebra package
}

\author{
MANuel Dubinsky, CÉSAR MASSRI, \\ ARIEL MolinueVo AND FEDERICO QUALlBRUnN
}

\begin{abstract}
In this article we present DiffAlg.m2, a differential algebra package for Macaulay2. It can perform the following operations: wedge products and exterior differentials of differential forms, contraction and Lie derivatives of differential forms with respect to a vector field and Lie brackets between vector fields.

Given a homogeneous differential operator of degree one $D$, the lack of an algebraic module structure attached to the kernel or image of $D$ hinders the study of $D$. The main purpose of DiffAlg.m2 is to handle these spaces degree-wise.
\end{abstract}

Motivation AND DESCRIPTION OF THE PACKAGE. Algebraic and differential operations arise naturally when working with differential forms and vector fields, e.g., wedge products and exterior differentials of differential forms, contraction and Lie derivatives of differential forms with respect to a vector field and Lie brackets between vector fields. Some important statements involving these operations include the following:

(a) A differential $r$-form $\omega$ in the affine space $\mathbb{K}^{n+1}$ descends to the projective space $\mathbb{P}_{\mathbb{K}}^{n}$ if it satisfies the equation

$$
i_{R} \omega=0,
$$

where $\mathbb{K}$ is a field, $R$ is the radial vector field $R=\sum x_{i} \frac{\partial}{\partial x_{i}}$ and $i_{R}$ denotes the contraction; see [Hartshorne 1977, Theorem 8.13, p. 176].

(b) If $\omega$ is a differential 1-form, then $\omega$ defines a foliation in $\mathbb{K}^{n+1}$ if it satisfies the Frobenius integrability condition given by the equation

$$
\omega \wedge d \omega=0
$$

see [Suwa 1995, Definition 2.2, p. 823].

The authors were supported by CONICET, Argentina.

MSC2010: 14-04, 53-04.

Keywords: differential operators, exterior algebra, vector fields.

DiffAlg.m2 version 1.5 
(c) Let $\omega$ be an integrable 1-form and $L_{X} \omega$ be the Lie derivative of $\omega$ with respect to a vector field $X$. Then, the solutions of the equation

$$
L_{X} \omega=0
$$

define all the infinitesimal automorphisms of the foliation given by $\omega$; see [Suwa 1995, Proposition 7.7, p. 845].

(d) Let $\omega$ be an integrable 1-form. Then the tangent space of the space of foliations at $\omega$ is given by the differential 1 -forms $\eta$ that satisfy the equation

$$
\omega \wedge d \eta+d \omega \wedge \eta=0
$$

see [Cukierman et al. 2009, Section 2.1. p. 709].

(e) Let $D$ be a bracket generating distribution. Some important invariants of $D$ are the ranks of the derived sequence

$$
a(p):=\operatorname{rank} D^{(p)}=\operatorname{rank}\left(D^{(p-1)}+\left[D, D^{(p-1)}\right]\right) ;
$$

see [Tanaka 1970, §1, pp. 8-9].

(f) A symplectic structure in a variety of dimension $2 r$ is given by a 2-form $\omega$ such that $d \omega=0$ and $\omega^{r} \neq 0$; see [Bryant et al. 1991, p. 41].

For a clear understanding of how DiffAlg.m2 deals with such equations, let us fix some notation.

Let $S=\mathbb{K}\left[x_{0}, \ldots, x_{n}\right]$ be the polynomial ring in $n+1$ variables and let $\Omega=$ $\bigoplus_{r \geq 0} \Omega^{r}$ be the exterior algebra of differential forms of $S$ over $\mathbb{K}$. Let $\Omega^{r}(d)$ denote the space of $r$-forms with polynomial coefficients of homogeneous degree $d$, where we assign degree 1 to each $x_{i}$ and degree 0 to each $d x_{i}$.

Therefore $\omega \in \Omega^{r}(d)$ can be written as

$$
\omega=\sum_{\substack{I \subset\{0, \ldots, n\} \\ \# I=r}} \sum_{\substack{\alpha \in \mathbb{N}^{n+1} \\|\alpha|=d}} a_{\alpha, I} x^{\alpha} d x_{I}, \quad a_{\alpha, I} \in \mathbb{K},
$$

where, for each $I$ of the form $I=\left\{i_{1}, \ldots, i_{r}\right\} \subset\{0, \ldots, n\}$, we let $d x_{I}$ denote $d x_{i_{1}} \wedge \cdots \wedge d x_{i_{r}}$ and for each $\alpha=\left(\alpha_{0}, \ldots, \alpha_{n}\right) \in \mathbb{N}^{n+1}$ we denote $|\alpha|:=\sum_{i=0}^{n} \alpha_{i}$ and $x^{\alpha}:=x_{0}^{\alpha_{0}} \ldots x_{n}^{\alpha_{n}}$.

Let $T$ be the module of vector fields with coefficients in $S$. Let $T(e)$ denote the homogeneous vector fields with polynomial coefficients of degree $e$, where we assign degree 1 to each $x_{i}$ and degree 0 to each $\frac{\partial}{\partial x_{i}}$ and, analogously to (1), $X \in T(e)$ can be written as

$$
X=\sum_{i=0}^{n} \sum_{\substack{\beta \in \mathbb{N}^{n+1} \\|\beta|=e}} b_{\beta, i} x^{\beta} \frac{\partial}{\partial x_{i}}, \quad b_{\beta, i} \in \mathbb{K} .
$$


Current algebraic software systems implement functionality to deal with differential forms and vector fields, but usually scalar parameters $a_{\alpha, I}$ and $b_{\beta, i}$ must be specified as fixed elements in $\mathbb{K}$. Instead, DiffAlg.m2 treats homogeneous forms and vector fields in a completely symbolic environment by considering the scalar coefficient rings

$$
\mathbb{K}\left[a_{\alpha, I}\right] \text { and } \mathbb{K}\left[b_{\beta, i}\right] \text {. }
$$

Scalar coefficients can be systematically obtained by looking at the coordinates of differential forms and vector fields written in the standard bases

$$
\mathcal{B}_{r, d}=\left\{x^{\alpha} d x_{I}\right\}_{\substack{\# I=r \\|\alpha|=d}} \text { and } \quad \mathcal{B}_{e}=\left\{x^{\beta} \frac{\partial}{\partial x_{i}}\right\}_{|\beta|=e}
$$

of the spaces $\Omega^{r}(d)$ and $T(e)$, respectively.

Importantly, when using DiffAlg.m2, each object is expected to be defined in its own coefficient ring. Then, certain operations, such as contraction or computing the wedge product, involve different input and output rings, producing a modification of the coefficients rings $\mathbb{K}\left[a_{\alpha, I}\right]$ or $\mathbb{K}\left[b_{\beta, i}\right]$. For greater clarity, consider the following example. Fix $\omega \in \Omega^{r}(d)$ and $X \in T(e)$ and consider the contraction $i_{X} \omega \in \Omega^{r-1}(d+e)$. Then, the following will be taking place:

\begin{tabular}{|l|ccc|}
\hline & $(\omega, X)$ & $\mapsto$ & $i_{X} \omega$ \\
\hline Ring & $\mathbb{K}\left[a_{\alpha, I}\right] \times \mathbb{K}\left[b_{\beta, i}\right]$ & & $\mathbb{K}\left[a_{\alpha, I}, b_{\beta, i}\right]$ \\
Basis & $\mathcal{B}_{r, d} \times \mathcal{B}_{e}$ & & $\mathcal{B}_{r-1, d+e}$ \\
\hline
\end{tabular}

As mentioned before, the main purpose of DiffAlg.m2 is to find algebraic solutions to equations in the context of differential algebra. Equations are treated differently in the linear and nonlinear cases:

(a) In the linear case, for example $i_{R} \omega=0$, DiffAlg.m2 can compute a basis of the solutions of the equation. Once this is done, it can also compute a generic linear combination of the elements of the basis; see Example 1.

(b) In the nonlinear case, for example $\omega \wedge d \omega=0$, the coordinates will be polynomial. In this case, DiffAlg.m2 would compute the ideal generating the space of solutions. This ideal can be obtained in two different ways: taking coordinates in the basis $\mathcal{B}_{r, d}$ or $\mathcal{B}_{e}$, or taking coordinates in the basis $\left\{d x_{I}\right\}$ or $\left\{\frac{\partial}{\partial x_{i}}\right\}$; see Examples 2 and 4 .

DiffAlg.m2 can also be a valuable tool for studying differential operators. The lack of algebraic theory to deal with such objects can be mitigated by nonconclusive computations easily made by DiffAlg.m2. As a first example, one could consider computing solutions of a differential operator degree-wise for low degrees. 


\section{SOME EXAMPLES.}

Example 1. In the following example we obtain a basis of the space of projective differential 2-forms in $\mathbb{P}_{\mathbb{K}}^{3}$. Then, we define a generic projective differential 2-form to be possibly used in further computations.

i1 : loadPackage "DiffAlg";

i2 : $\mathrm{R}=$ radial 3

$02=\mathrm{x}_{0} \mathrm{ax}+\mathrm{x}_{1} \mathrm{ax}+\mathrm{x}_{2} \mathrm{ax}+\mathrm{x}_{3} \mathrm{ax}$

02 : DiffAlgField

i3 : w = newForm $(3,2,1, " \mathrm{a} ")$;

$03=(a x+a x+a x+a x) d x d x+(a x+a x+a x+a x) d x d x$

$+\left(a_{30} x_{91}+a_{152} x_{21}+a_{3} x_{1} x_{2}+a_{2} x_{0}+a_{8} x_{1}+a_{14} x_{2}+\right.$ $\left.a_{20} x_{3}\right) d x_{0} d x_{3}+\left(a_{40}+a_{10} x_{1}+a_{16} x_{2}+a_{22} x_{3}\right) d_{1} d_{3}+\left(a_{5} x_{0}+a_{11} x_{1}+\right.$ $\left.a_{172} x_{23}+a_{3} x_{2}\right) d x$

०3 : DiffAlgForm

i4 : pretty ring $\mathrm{w}$

$$
\begin{aligned}
& 04=\frac{Q Q[i]}{i^{2}+1}\left[a_{0}, a_{1}, a_{2}, a_{3}, a_{4}, a_{5}, a_{6}, a_{7}, a_{8}, a_{9}, a_{10}, a_{11}, a_{12}\right. \text {, } \\
& \left.\mathrm{a}_{13}, \mathrm{a}_{14}, \mathrm{a}_{15}, \mathrm{a}_{16}, \mathrm{a}_{17}, \mathrm{a}_{18}, \mathrm{a}_{19}, \mathrm{a}_{20}, \mathrm{a}_{21}, \mathrm{a}_{22}, \mathrm{a}_{23}\right]\left[\mathrm{x}_{0}, \mathrm{x}_{1}\right. \text {, } \\
& \left.\mathrm{x}_{2}, \mathrm{x}_{3}\right]\left[\mathrm{dx}_{0}, \mathrm{dx}_{1}, \mathrm{dx}, \mathrm{dx}_{3}\right]
\end{aligned}
$$

i5: $\mathrm{K}=\operatorname{genKer}(\mathrm{R}-\mathrm{w}, \mathrm{w})$;

i6 : length $\mathrm{K}$

$06=4$

i7 : v = linearComb (K, "a")

$$
\begin{aligned}
& 07=\left(a_{0} x_{1}-a_{3} x_{0}\right) d x_{1} d x_{0}+\left(-a_{0} x_{2}+a_{3} x_{0}\right) d x_{2} d x_{0}+\left(a_{0} x_{3}+a_{3} x_{1}\right) d x d x+ \\
& \left(a_{1} x_{2}-a_{2} x_{0}\right) d x_{3} d x+\left(-a x_{0}-a_{32} x_{2}\right) d x_{3} d x+\left(x_{0}+a_{1} x_{2}\right) d x d x
\end{aligned}
$$

07 : DiffAlgForm

i8 : pretty ring $\mathrm{v}$

$$
08=\frac{Q Q[i]}{2}+\left[\begin{array}{l}
2 \\
i
\end{array}\right.
$$


Let us explain part of the code:

i2. Creates the radial vector field in four variables. We are denoting the basic field $\partial / \partial x_{i}$ as $a x \_i$.

i3. Creates a generic linear 2-form in $\Omega_{\mathbb{K}^{4}}^{2}(1)$, with the coefficients indexed as $a_{i}$.

i4. Shows the ring of definition of $w$.

i5. Gets a basis (as a Macaulay2 list) of forms in $\Omega_{\mathbb{K}^{4}}^{2}(1)$ that descend to projective space. The operation $R \_w$ computes the contraction of the differential form $w$ with the vector field $R$.

i6. Gets the dimension of $\Omega_{\mathbb{P}^{3}}^{2}(1)$ in projective 3-space.

i7. Defines a generic projective form with coefficients $a_{i}$.

i8. Shows the ring of definition of $v$.

Example 2. In the finite-dimensional $\mathbb{K}$-vector space $\Omega^{1}(d)$, the solutions of the equation $\omega \wedge d \omega=0$ determine an algebraic variety; its points are the integrable differential 1 -forms of degree $d$. In the following example, we compute the equations of the variety of integrable 1 -forms of degree 1 in 3 -dimensional space.

It is worth mentioning that, for $n \geq 3$ and $d>5$, it is an open problem to classify the irreducible components of this varieties; see [Cukierman et al. 2009].

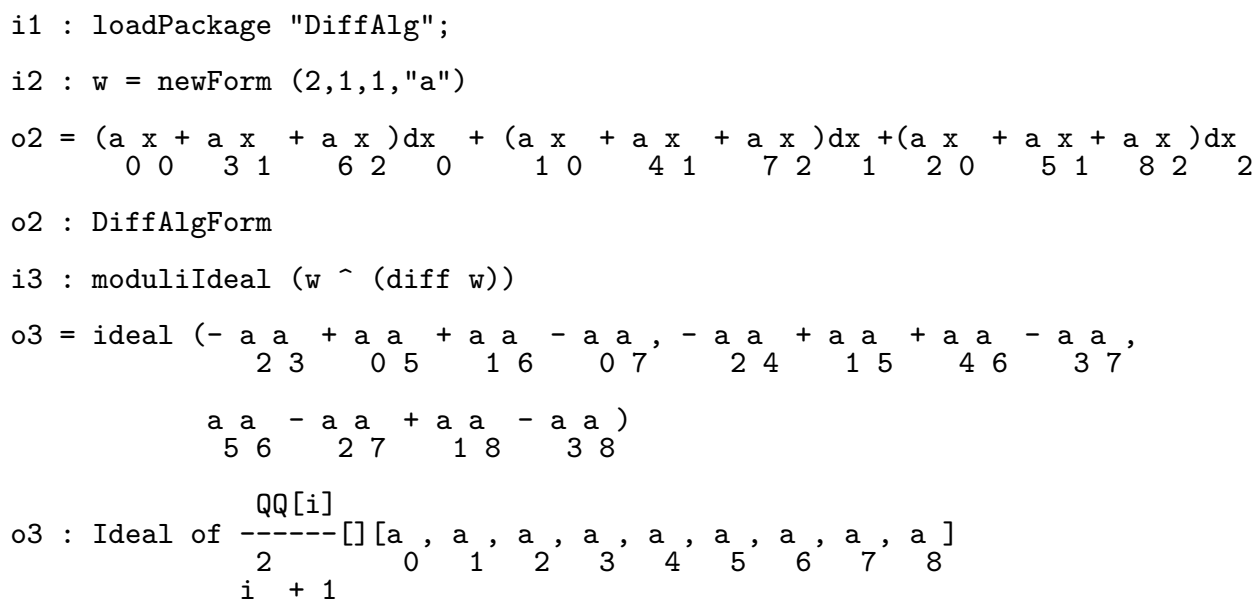

About the code:

i2. Creates a generic linear 1 -form in $\Omega_{\mathbb{R}^{3}}^{1}(1)$, with coefficients $a_{i}$.

i3. Returns the ideal of the scalar coefficients given by $w \wedge d w=0$.

Example 3. Let $D$ be a 2-dimensional distribution generated by vector fields $X$ and $Y$ in 5-dimensional space. In the following example we compute the ranks of the derived distributions $D^{(p)}$. We verify that this derived series eventually spans 
the entire tangent space. A distribution $D$ satisfying this condition is called bracketgenerating.

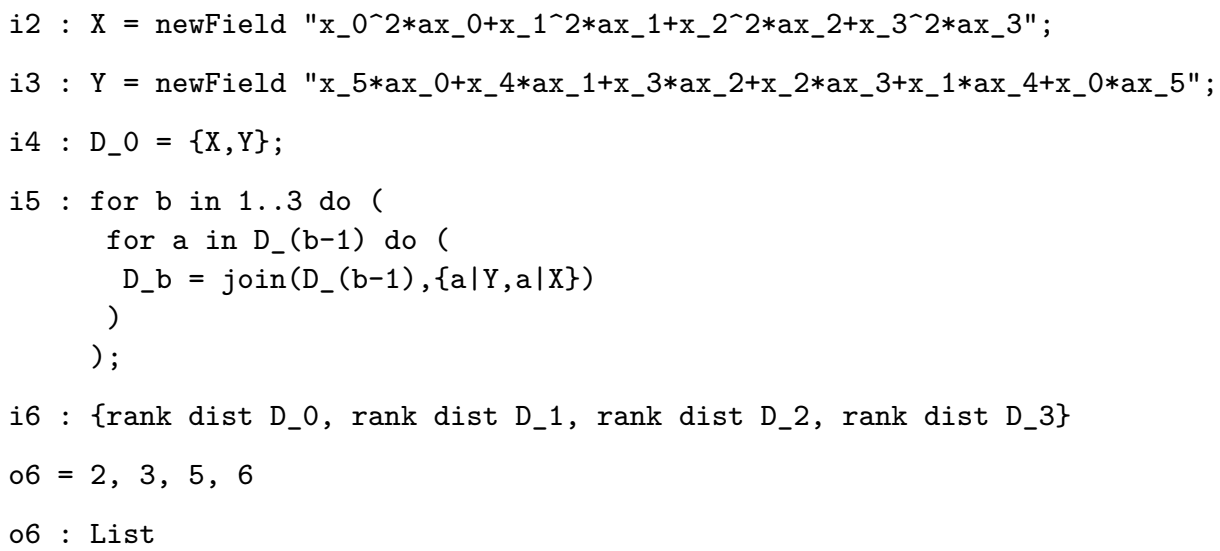

About the code:

i5. Computes the derived sequence.

i6. Prints the ranks of the derived series.

Example 4. In the following example, we generate a random rational 1-form of type $(1,2)$ in $\mathbb{P}_{\mathbb{K}}^{2}$. First, we compute (the dimension of) the space of its integrating factors; see [Suwa 1995, pp. 828-829]. Then, we compute the ideal of its singular locus (the ideal where it vanishes).

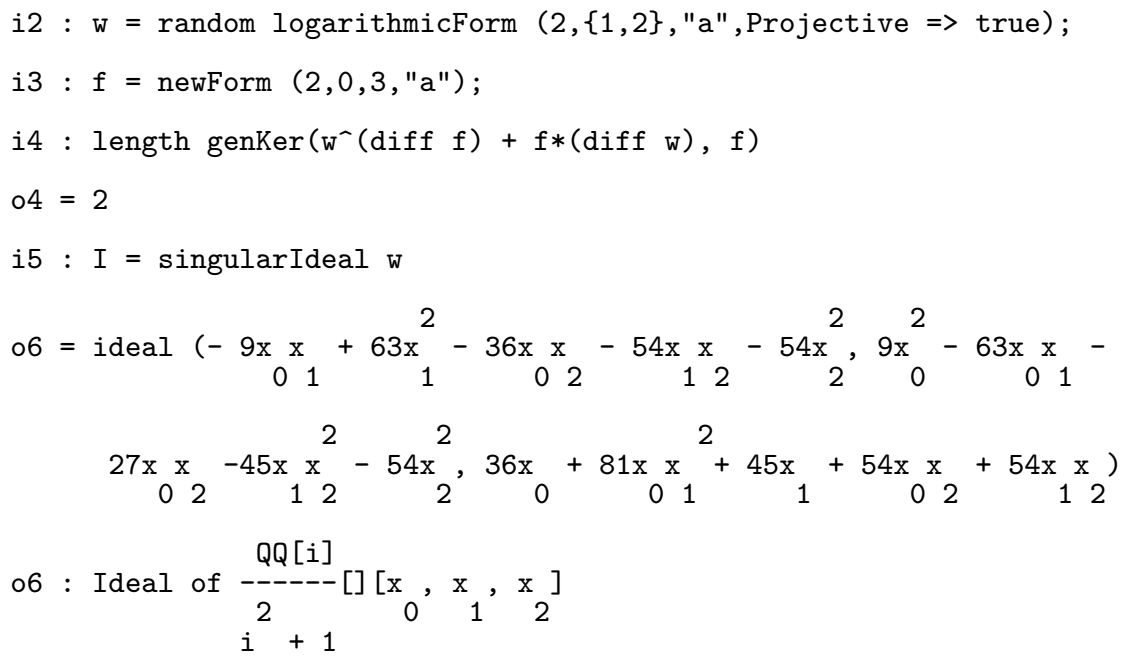

SuPPLEMENT. The online supplement contains version 1.5 of DiffAlg.m2. 


\section{REFERENCES.}

[Bryant et al. 1991] R. L. Bryant, S. S. Chern, R. B. Gardner, H. L. Goldschmidt, and P. A. Griffiths, Exterior differential systems, Mathematical Sciences Research Institute Publications 18, Springer, 1991. MR Zbl

[Cukierman et al. 2009] F. Cukierman, J. V. Pereira, and I. Vainsencher, "Stability of foliations induced by rational maps", Ann. Fac. Sci. Toulouse Math. (6) 18:4 (2009), 685-715. MR Zbl

[Hartshorne 1977] R. Hartshorne, Algebraic geometry, Graduate Texts in Mathematics 52, Springer, 1977. MR Zbl

[Suwa 1995] T. Suwa, "Unfoldings of codimension one complex analytic foliation singularities", pp. 817-865 in Singularity theory ((Trieste, 1991)), edited by D. T. Lê et al., World Sci. Publ., River Edge, NJ, 1995. MR Zbl

[Tanaka 1970] N. Tanaka, "On differential systems, graded Lie algebras and pseudogroups", J. Math. Kyoto Univ. 10 (1970), 1-82. MR

Received: 27 May 2015 Revised: 26 Oct 2018 ACCEPTED: 19 Nov 2018

MANUEL DUBINSKY:

manudubinsky@gmail.com

Departamento de Computación, Universidad de Avellaneda, Avellaneda, Argentina

CÉSAR MASSRI:

cmassri@caece.edu.ar

Departamento de Matemática, Universidad CAECE, Buenos Aires, Argentina

Ariel Molinuevo:

amoli@dm.uba.ar

Instituto de Matemática, Universidade Federal do Rio de Janeiro, Rio de Janeiro, Brazil

FEDERICO QUALLBRUNN:

fquallb@dm.uba.ar

Departamento de Matemática, Universidad de Buenos Aires, Pabellón I, Ciudad Universitaria, Buenos Aires, Argentina 

Strongly stable ideals and Hilbert polynomials

Davide Alberelli and Paolo Lella

DiffAlg: a Differential algebra package

Manuel Dubinsky, César Massri, Ariel Molinuevo and Federico Quallbrunn

Matroids: a Macaulay2 package

Justin Chen

Computing quasidegrees of A-graded modules

Roberto Barrera

An algorithm for enumerating difference sets

Dylan Peifer

Hyperplane arrangements in $\mathrm{CoCoA}$

Elisa Palezzato and Michele Torielli

Numerical implicitization

Justin Chen and Joe Kileel

Random Monomial Ideals: a Macaulay2 package

Sonja Petrović, Despina Stasi and Dane Wilburne

Ben Drabkin, Eloísa Grifo, Alexandra Seceleanu and Branden Stone

The gfanlib interface in Singular and its applications

Anders Jensen, Yue Ren and Hans Schönemann 